\section{THE EFFECTS OF RESPIRATION ON THE CIRCULATION;}

AND

\section{THE PULSUS PARADOXUS VEL PULSUS INSPIRATIONE INTERMITTENS.} $\mathrm{BY}$

Sir JAMES BARR, M D , LL.D , F.R.C.P., F.R.S.E.,

SENIOR PHYSICIAN, LIVERPOOL ROYAL INFIRMARY ; CHAIRMAN OF 'THE MEDICAL FACULTY, LIVEKPOOL UNIVERSITY': PHKSIDENT OF THE LANCASHIRE AND CHESHIRE BRANCH OP THE BRITISH MEDICAL ASSOCIATION.

Dr. P. Watson Williams, in his letter ${ }^{1}$ on the pulsus Jaradoxus, has drawn attention to a difference of opinion between Yrofessor Wenckehach and myself as to the explanation of this phenomenon. Many years ago I ettled the question of the pulsus paradoxus to my own satisfaction, and as I have lately given over missionary work it does not trouble me very much whether Wenckebach or anyone else agrees or disagrees with me. It seems to me that there is no use trying to settle anything in medicine, because no cooner does anything appear settled than up springs some one with a new theorv, or an old theory in a new garb. Therefore, Professor Wenckebach's article on adherent pericardium, ${ }^{2}$ which I read with much interest, did not draw me from my lair; nor would the letter of my friend Dr. Watson Williams in the JournaL have done so, but a private letter from Dr. Williams requesting $\mathrm{my}$ criticism forces me into the arena.

I have again carefully read Professor Wenckebach's paper, ${ }^{2}$ and I should not care to speak of the pulse in the case which he describes as the pulsus paradoxus, but I may be quite reasonably told that Wenckebach felt the pulse and thus designated it, and surely such an authority on the pulse should be quite good enough for me or any one else. However, I am quite willing to approach the subject under an aspersion of medical treason for daring to question the observations, or rather deductions from observations, of this undoubtedly great authority. It does not matter much what a man says so long as you understand what he means, so let us examine his word picture and illustrations.

The pulse was small, weak, and frequent, diminishing during inspiration (pulsus paradoxus); the veins were considerably overfilled, and during inspiration there was marked swelling of the veins in the neck.

He does not say whether the diminution in the pulse during inspiration was in frequency or force, or both, but in the pulse tracings taken at a later date there is slight diminution in frequency but no alteration in the character of the pulse. There are very much greater respiratory variations in $\mathrm{my}$ own pulse, and in the pulse of every healthy individual with a good respiratory pump. I call my own palse normal; I might call that of Professor Wenckebach's patient abnormal, but there is no reason why I should call it a pulsus paradoxus unless it were for the mere sake of agreeing with Professor Wenckebach.

The fact of the matter is Kussmaul first described the pulse under the title pulsus paradorus in a case of chronic indurative mediastinitis, and thought that it was pathognomonic of the disease, and since then every patient with extensive pericardial adhesions has got to have a pulsus paradoxus whether he likes it or not. It was soon found that many persons, both in health and disease, had this form of pulse, hence it ceased to be pathognomonic of mediastinitis. Under whatever conditions it occurred, it was looked upon as an abnormality, something to write about; and it seems to have had a peculiar fascination for observers, especially of the juvenile type (among which I certainly do not include Professor Wenckebach, as he is one of the keenest and most accurate observers I know), and hence an enormous amount of printer's ink and paper have been wasted over one of the commonest of cardiac phenomena. I should like to say with Prior, "Let him be kept from paper, pen, and ink, So may he cease to write, and learn to think."

Each writer on this subject has had almost as many the ories as the number of cas 3 he has described. For my own part, I have always accepted the dictum of
Sir Ieaac Newton that we are to admit no more canses of natural things than such as are both true and sufficient to explain their appearance, hence the theory which I have on many occasions put forward is intended to explain every case; but before I deal with my own views I must continue my destructive criticism of others.

When Kussmaul ${ }^{8}$ published his cases of indurative mediastino-pericarditis in 1873, he looked upon the pulsus paradoxus as pathognomonic of the disease, and explained the phenomenon by suggesting that the inspiratory act put a strain on the adhesions which kinked the aorta or great vessels, and thus prevented the pulse from reaching the wrist. This absurd explanation was generally accepted in Germany and in this country with unreasoning credulity on account of its distinguished author; but how any one who had the slightest knowledge of natural laws could propound or accept such a theory is a puzzle to my mind. Nowadays it is the heart rather than the great vessels which is hampered by the adhesions, and writers talk of the adhesions dragging in the chest walls. I wonder if these writers have forgotten that there is such a thing as atmospheric pressure. When a child of very tender age is suffering from laryngeal diphtheria and the chest walls are caved in at every attemprs at inspiration no one would say that they are retracted by the membrane in the larynx and trachea; but it has just got as much or more to do with the falling in of the chest walls than pericardial and pleuritic adhesions have. During inspiration, and when the heart contracts and alters its shape, there is the formation, or the tendency to the formation, of a partial vacuum, and if this cannot be filled up by the contents of the thorax, the chest walls are driven in by the difference between the atmospheric pressure inside and outside the thorax.

If the chest wall were retracted by fibrous adhesions between the heart and itself, there must be some fulcrum from which the heart can act, and some writers have apparently recognized this, as they have supposed the heart also to be anchored to the posterior mediastinum, or even to the vertebral column. In the majority of cases there is no such universal adhesione, and when they do occur the heart's action is very weak, the force of even the left ventricular systole rarely equalling a fluid pressure of $2 \mathrm{lb}$. to the square inch, which is a trivial force compared with that of the atmosphere. When the heart acts from its attached base as a fulcrum it might readily through some adhesions retract the intercostal spaces, but such force is not likely to retract the bony chest walls. The systolic "retraction" and diastolic recoil of the lower part of the sternum and costal cartilages, which frequent]y occur in cases of pericardial adhesions, are due to the pressure of the atmosphere.

Even Wenckebach attributes all these phenomena to the heart and its adhesions, and takes no notice of such a powerful and ever-present force as the atmosphere, as the following sentences from his paper will show ${ }^{2}$ :

Indeed, the heart is hindered in its lateral forward movements by tough and hard fibrous masses, so that it can neither be properly filled nor properly emptied. By its shortening at every systole it pulls the chest wall in front, and by means of the posterior mediastinum, at the vertebral column. Neither of these structures gives way. In youthful individuals the ribs may be so flexible that the heart is able to pull them inward ; in older patients this will be impossible; and in both cases the heart has opposed to it an enormous amount of extra work.

The heart is more likely to be anchored to the chest wall than the chest wall to be pulled in by the heart: moreover, the exocardial adhesions are always sufficiently long to allow of cardiac systole.

The diaphragm is greatly limited in its movements; thus, the abdomen will not be pushed forward during inspiration, or very slightly. At that same moment the diaphragm pulls, by means of the heart, at the chest wall in the regio cordis, so there is no forward movement of the chest, but rather an inspiratory retraction of the chest.

One would think that the pull would depend on the amount of movement, yet, according to this fanciful explanation, a greatly limited movement of the diaphragm retracts the chest wall through the pericardial adhesions of the heart to the diaphragm and chest walls. According to this authority the heart pulls one way and the diaphragm another, and the resultant is what 
happens; this, it is presumed, should satisfy any one who has got a mathematical mind!

The inspiratory traction on the heart, which cannot give way [it was previously the chest wall which could not give way, now it is the heart], is bound to affect injuriously the action of this organ. The "deblt" of the heart, too small in expiration must become still smaller during inspiration. $\Delta s$ a result of this embarrassing action during inspiration, the wellknown symptom of pulsus parsdoxus is produced-nsmely, a lessening of the blood pressure and of the pulse wave in the arterial system. Another symptom furnishes further proof that during inspiration the heart is working under worse conditions-namely, the inspiratory swelling of the veins of the neck. In normal conditions these veins are emptied by the increased suction of the thoracic cavity during inspiration. Here the contrary happens; the flow of blood to the heart is diminished. We may pat aside the more subtle question whether there be a direct traction on the aorta or on the large veins, as some authors assume. The more embarrassed condition of the hesrt during inspiration is obvious, and both symptoms-the pulsus paradoxus and the swelling of the veins -are coupled together and have the same origin. Perhaps it is noteworthy that in the patient here described both symptoms were present; in another patient I saw both were absent.

This explanation, like the respiration in these cases, is very laboured. It would seem, according to this, that, under similar conditions, the symptoms may be present or may be absent.

He then quotes my explanation of the pulsus paradoxus from my Toronto address, ${ }^{4}$ On the Circulation Viewed from the Periphery, but he omits a rather important point, in my opinion, the storage of blood in the lungs; and he disposes of my views as follows :

A great deal might be said about this assertion. Certainly the explanation by an increased suction of the chest (be it working on the arteries or on the veins) holds good in a great many cases, distinctly where there are a low arterial pressure, a weak heart action, and strong inspiratory movements. But it does not hold good in the cases of adherent pericardium. The particular consequence of this disease is the impediment to inspiration, not by narrowing of the air tract, but by the anchoring of the diaphragm. There is not an increased fall of pressure in the chest, but a very deficient inspiratory mechanism And the proof that there is not an increased suction of the chest and not a sudden emptying of the veins is shown by the inspiratory swelling of the veins. So we have to adopt the explanation of an increased hindrance to the circulation during inspiration; and I venture to think that the increase of the hindrance is due to the tugging of the diaphragm on the heart, that cannot give way on account of its being fixed to its immovable surroundings.

We find him acknowledging that my theory, or as he calls it assertion, is correct in a great many cases, and my contention is that any true theory which explains any one case should be comprehensive enough to explain every case, but Professor Wenckebach, while agreeing that the pulsus paradoxus may be associated with a strong negative pressure within the thorax, yet contends that in his case it occurred with a positive pressure sufficient to obstruct the entrance of blood. You might as well expect the experiments of Müller and Valsalva to have the same effects. It might be worth Professor Wenckebach's while to consider whether the pulse in his case should be dubbed the pulsus paradoxus, and whether this supposed tugging of the diaphragm really existed; and, if so, what effects had it, if any?

Before giving my own views, it might be well to state what seems to me the evolutional history of this subject.

In 1740 Valsalva found that after a deep inspiration, if you close the glottis, and make a most forcible expiratory effort, the circulation is arrested.

In 1799 Dr. Wilson ${ }^{5}$ of Newcastle held that the blood in the veins was returned to the heart by atmospheric pressure-a doctrine, said Haller, not to be despised.

In 1815 Dr. James Carson ${ }^{5}$ of Liverpool taught that great assistance was rendered to the circulation by the aspirating effect of the thorax during inspiration sucking in the blood from the systemic veins. This theory was afterwards further elaborated by Dr. Andrew Buchanan of Glasgow, Dr. Patrick Black of London, and Dr. Leonard Hill, and it has now become an established fact in phygiology. Dr. Carson also seems to have accepted the view of Dr. Brown Langrish that the heart dilated independently of the force of the returning current.

In 1826 Dr. David Barry ${ }^{\circ}$ published two important essays, on the motion of the blood in the veins, and on external absorption. This experimental investigation corroborated the work of Carson, and well exemplified the part played by the suction of the thorax and the atmospheric pressure in carrying on the circulation in the veins. Dr. Barry's work received the high approval of Baron Cuvier and Professors Dumbril and Laënnec. He turned his experiments to practical account by showing that the application of a powerful cupping glass to a recentlypoisoned wound would prevent the absorption of the poisonous matter.

- In 1838 Johannes Müller noted that if after \& deep expiration you close the glottis and make a very forcible inspiratory effort the pulse at the wrist disappears, and this he ascribed to the engorgement of the pulmonary vessels and the consequent emptiness of the left ventricle. This is the very opposite of Valsalva's experiment, though in both you can obliterate the pulse at the wrist; but in the latter the artery remains full, whereas in Müller's experiment it is empty.

In 1850 Dr. C. J. B. Williams ${ }^{7}$ stated that:

Advanced stages of severe pericarditis and adhesions of the pericardium present a remarkable kind of inequality, and even intermission, in the radial pulse, whilst the heart's pulsations are quite regular; in fact, some of the latter are too weak to reach the wrist, hence the irregularity ; and I have distinctly ascertained that the weak or deficient beats are those corresponding with inspiration, whilst the stronger pulses are those enforced by the expiratory act, which thus helps the weak heart and augments its propulsive power. So it often happens that the act of coughing gives a momentary strength to a weak pulse.

In 1854 , at the Tübingen Clinic, Griesinger ${ }^{8}$ had a case of fibrinous mediastinitis under his care, in which he noticed

" a small, irregular pulse, while the heart beat regularly; the intermissions of the pulse occurred at regular intervals and simultaneously with inspiration." At theautopsy, "stiff, stringy exudations were found in the connective tissue in which the large vessels are imbedded after their exit from the pericardium. Some of the venous trunks were notably constricted, as was also the aorta in its ascending portion and arch; it was also indented and partly twisted about its axis. The pericardial cavity was almost obliterated by fibrinous membranes."

In 1864 Friedreich ${ }^{9}$ described a sudden diastolic collapse, and a systolic filling of the cervical veins which frequently occur in cases of mediastinitis.

In $1873 \mathrm{Kussmaul}^{3}$ described the pulsus inspiratione intermittens or pulsus paradoxus in association with indurated mediastino-pericarditis of which he thought the pulse pathognomonic. He also noticed the inspiratory swelling and diastolic subsidence of the cervical veins in these cases. He abscribed the swelling of the veins and lessened pulse to the same cause, namely, the fibrous bands kinking or obstructing the great vesselsarteries and veins-during inspiration. We have seen how Wenckebach also ascribes the two phenomena to the same cause, but he places the obstruction a little further back, namely, in the heart.

In 1874 Traube and Baeumler ${ }^{10}$ found the pulsus paradoxus in cases of pericarditis exsudativa without mediastinitis, but in these cases the inspiratory swelling and expiratory collapse of the cervical veins did not occur.

In 1876 Josef Bauer wrote an excellent article on diseases of the pericardium in von Ziemssen's Cyclopaedia. He refers to cases of purulent pericarditis and effusion into the left pleura in which the pulsus paradoxus occurred. He gives the following explanation of the phenomenon :

In my opinion the inspiratory displacement of the thorax may produce, under different conditions, mechanical obstructions which result in the expulsion of a less amount of blood these may occur in the aorta, perhaps also in the subclavian or at the origin of the vessels, and then the heart sounds are unchanged; or they may be in the heart itself, and then we find at the same time a diminution of its action. The production is favoured by a weak, degenerated heart. Besides these mechanical hindrances in the circulatory system, caused by the act of inspiration, the phenomenon of the pulsus inspirstione intermittens may also be produced by obstruction to the entrance of air into the lungs, for the inspiratory augmenta. tion of the negative pressure (suction) within the thorax opposes the supply of a proper amount to the aortic system. In this case there is an exaggeration of a normal relation, for even under normal conditions the sphygmograph shows that the negative pressure within the thorax during inspiration can cause a slight diminution of the wave, as Riegel and Sommerbrodt have shown.

When dealing with the retraction of the chest walls in 
cases of pericardial adhesions, he is about the only author I have met who has not neglected the atmospheric pressure.

In $1877^{11}$ and $1882^{12}$ I published papers on Reduplica. tion of the Cardiac Sounds; in $1884^{13}$ on the Causes and Mechanism of the Cardiac Impulse; and in 1900 and $1901^{14}$ on the pulsus paradoxus, in all of which I entered very fully into the effects of respiration on the circulation.

In 1877 Stricker ${ }^{15}$ published a case of the pulsus paradoxus in tuberculous pericarditis without mediastinitis; in 1883 the occurrence of the pulsus paradoxus was noted in a case of purulent pericarditis by $\mathrm{J}$. West, ${ }^{16}$ and in a case of pericarditis without mediastinitis by E. Boehr. ${ }^{17}$

In 1893 Dr. E. M. Brockbank ${ }^{18}$ found this phenomenon in a case of acute laryngitis.

In 1894 and 1896 the late Dr. Thomas Harris made some interesting communications in the Medical Chronicle on indurative mediastino-pericarditis, in which he referred to the occurrence of the pulsus paradoxus. In the Lancet of April 22nd, 1899, he published a valuable article on the pulsus paradoxus, with special reference to its occurrence on one side only.

In 1897 Gerhardt ${ }^{19}$ published two cases in which the phenomenon was only observed in one radial artery, and he ascribed the cause to variations in the size of the two subclavian arteries, but in Dr. Harris's unilateral case no such difference was found. In connexion with such unilateral variation, I may mention a little trick, taught me by Mr. C. E. Walker (of cancer fame), of abolishing the pulse in one or both upper extremities by simply com pressing the subclavian artery as it passes over the first rib by the clavicle. Some such compression, voluntary or involuntary, during inspiration would readily account for a unilateral pulsus paradoxus in the upper extremities; but, of course, the other arteries could not be thus affected. However, in most pulse records the observers seem to think it unnecessary to refer to any artery except the radial.

There are a plethora of articles on this subject to which any further reference would serve no useful purpose.

I now come to the letter of Dr. Watson Williams, ${ }^{1}$ and at the very outset I must fall foul of $\mathrm{my}$ friend for ascribing a statement to Wenckebach about myself which he does not make :

He (Wenckebach) shows that the explanation of pulsus paradoxus by Sir James Barr and the majority of clinicians ... does not hold good in adherent pericardium.

I thought my views were my own, but it seems that I have got to be lumped with others who have got no personal theories, but who have swallowed the physical absurdities of Kussmaul and others, and who, with the exception of Bauer, have utterly ignored the potent force of atmospheric pressure. He fairly sums up Wenckebach's criticism of my views as applied to cases of adherent pericardium:

There is not an increased fall of venous pressure, there is not an increased suction of the chest, and there is not a sudden emptying of the veins, so we have to adopt the explanation of an increased hindrance to the circulation during inspiration.

I at once challenge Wenckebach's facts. In the vast majority of cases of adherent pericardium, during inspiration there is a distinct fall in the intrathoracic pressure both in the aërial and blood-vascular cavities; the respiratory pump is in action and the veins of the neck shrink. These things may not have happened in Professor Wenckebach's case, but then I maintain that there was no pulsus paradoxus, and he has given no evidence to show that there was, and in another case he acknowledged that there was no inspiratory swelling of the cervical veins. He has no right whatever to reason from a particular case to a general. His reasoning would just be as good if he said, "Man is an animal ; therefore all animals are men." I happen to have 3 cases of mediastino.pericarditis with pleuritic adhesions under my care at present, and not one of them has got the pulsus paradoxus. In two of the cases the veins of the neck collapse during inspiration, and in the third there is just an appreciable diminution in the veins of the neck (which are constantly swollen), at the very beginning of inspiration, and then they fill up during the remainder of the period. This case is almost an exact counterpart of Professor Wenckebach's case.
There are extensive pericardial adhesions, adhesions of both pleurae, especially the left, general venous obstruction, distended cervical veins; scarcely any movement of the chest walls, and any expansion during inspiration is counterbalanced by the atmospheric pressure caving in the left side of the chest; a very large, hard liver, ascites for which he has been tapped three times, and for the present, at least, the fluid has disappeared under the influence of adrenalin. This boy has been under observation for some years, and I rather think was seen by Professor Wenckebach when he visited my wards. In the early bistory of this case fluid was drawn from both pleurae, and his pericardium was tapped twice, 19 and $20 \mathrm{oz}$. of fluid respectively being drawn off. On the second occasion I nearly killed him by the injection of 40 minims of adrenalin solution $(1$ in 1,000$)$ into the pericardium. This turned out to be too large a dose for the pericardium, but as this was the first time that the injection was ever performed, I had no previous experience to guide me, except the effect on the pleura. However, the immediate injection of nitro-glycerine and atropine brought him round.

If there be little or no variations in the thorax during respiration, how can you expect respiratory variations in the pulse? If the respiratory pump is out of gear, I for one do not expect it to act, but it is generally thought that a case of mediastino.pericarditis should have a pulsus paradoxus, and hence every little irregularity in the pulse is thus dubbed. If the chest is blown out with large emphysematous lungs and perhaps adherent pleurae, the intrathoracic pressure raised, and scarcely any fall in the pressure during inspiration, there can be practically no respiratory assistance to the circulation, and little or no inspiratory effect on the pulse. Dr. Watson Williams ${ }^{20}$ suggests

that the dominant factor in the production of pulsus paradoxus is the physiological rhythmical variation in the activity of the cardio-inhibitory centre, corresponding to the rhythmical variation in, and activity of, the respiratory centre.

Dr. Williams is a bold man to talk of the cardioinhibitory centre in these days of the myogenic function of the heart, muscular production of a contraction stimulus and its conduction, irritability, contractility, rhythmicity, not forgetting the bundle of His. Although I think the heart would get on very badly without its nerves, yet I think the time element is strongly against any such theory as a cause of the pulsus paradoxus. We believe that the knee-jerk is not a reflex movement, because the response is far more rapid that any nerve reflex. In the case of the heart the reflex is much slower than that travelling along the voluntary nerves, but the response of the pulse to inspiration is even quicker than the kneejerk. The atmospheric pressure; on which I contend this phenomenon depends, is an ever-present force, and its effect is practically instantaneous with the causes which induce the variations in pressure, as is readily shown in sphygmographic tracings taken during the various phases of respiration.. When a deep inspiration is started at the end of the cardiac cycle the next cycle is lengthened, and the arterial blood pressure falls; these phenomena could not possibly occur so quickly if they were determined by any organic reflex. The effects of respiration on the pulse are well illustrated in my paper on reduplication of the cardiac sounds, published in the Liverpool Medico-Chirurgical Journal, July, 1882. That number of the journal is now out of print, but I shall be pleased to send a copy of the paper to any one who desires it.

I am now in a position to explain my own views on the causation of the pulsus inspiratione intermittens, and by way of illustration I shall first start with the experiments of Valsalva and Müller.

The experiment of Valsalva carried out in 1740 has been kept alive by the records of physiologists, but I do not know one of them who has tested its accuracy. The aim of this experiment is not merely to stop the pulse at the wrist, but also to arrest the heart's action, and the ordinary Englishman is so cautious that if you ask him to try it, he wishes first to see how it pans out on yourself, and if he happen to think that your congested face betoken any risk of an apoplectic seizure he begs to be excused, as he has got a wife and family, or is going to get some encumbrance, but he never wishes you to think that there is any personal apprehension. However, I have carried out this experiment sufficiently often on myself (and I was the 
first on whom I tried it) and on other medical men and students to warrant me not only in corroborating the accuracy of Valsalva's observation, but also in giving the following refinements on the experiment.

When a person with a good respiratory pump takes a deep inspiration, and then tries to expire forcibly without allowing any air to esc ipe, he produces an intrapulmonary pressure of 80 to $100 \mathrm{~mm}$. of mercury. I make the individual who is carrying out the experiment, after taking a deep inspiration, blow into a mercurial manometer without, of course, allowing any of the air to escape. You can thus see what the intrapulmonary pressure is, and how it is sustained, and there can be no faking of the experiment. During the deep inspiration the heart and lungs are well supplied with blood, the blood pressure in the pulmonic and arterial systems falls, the cardiac contractions are effective and slightly quickened, and the heart sounds are clear. During the compression period no more blood enters the chest, as the intrathoracic pressure is much higher than that in the extrathoracic veins. At first the heart is well supplied with blood-on the left side from the large lung reservoir, and on the right side from the superior vena cava and its immediate tributaries, and also from the inferior vena cava and liver, as during the compression of the chest the abdomen is submitted to a similar pressure through the action of the diaphragm and the abdominal muscles. Therelore there is practically no alteration in the force of the cardiac contraction or in the lengths of the cycles until the surplus blood in the two reservoirs has been disposed of, or there may be slight slowing of the heart, owing to the increased blood pressure in the pulmonic and systemic systems, and the pulse becomes smaller and firmer When the complementary and tidal blood have been disposed of, which is generally accomplished in four or five beats of the heart, the pulse gradually disappears in all the superficial arteries, but the vessels remain fall, and the heart's sounds cease. The pulse and heart sounds may remain absent as long as the patient is able to keep up the pressure, and they are generally absent in any successful experiment for 8 or 10 seconds. It would seem to the observer that the heart had actually come to a standstill but when the experiment is viewed under the light of the $x$ rays the heart is seen to keep up a slow rhythmical contraction, getting gradually smaller in all its dimensions as it disposes of the supplementary blood in the thorax; and, finally, it does not appear half the size that it did during inspiration. In its contracted state the range of movement is so slight as to be barely appreciable. The heart is best viewed from the back, and the left side is seen to contract until it comes close to the border of the dark shadow of the spine and sternum. When the pressure is relaxed and an inspiration taken, the heart fills up immediately and resumes its wide range of movements. The experiment is most success-

fully carried out on a person with a good respiratory pump and low blood pressure, and the whole experiment barely occupies half a minute. During the compression period the extrathoracic venous pressure is gradually rising, and it is probable that the pressure might rise so high (especially if the experiment was only partially successful) as to force the blood in against an intrathoracic pressure of, say, 40 to $50 \mathrm{~mm}$. of mereury, more especially if this intrathoracic pressure was intermittent or not well sustained. In this case the pulse may not quite stop or only for a beat or two, and the pressure may rise sufficiently in the right ventricle to give rise to a tricuspid systolic murmur; this murmur disappears when the normal circulation is re-established. In such a case I have seen the heart's action much quickened towards the end of the experiment, so it was evident that some blood was getting into the chest either from the systemic venous pressure being so high as to force the blood in, or the intrathoracic pressure being either intermittent or not sufficiently high to keep the blood out. In cases of very high arterial tension the experiment is difficult to successfully carry out owing to the powerlul left ventricle forcing the blood onwards, but even in these cases a powerful chest is able to shut the hlood out, and so make the experiment a success. At the very instant you relieve the intrapulmonary pressure the heart is filled up, and restarts its usual beat, and the pulse returns; the arteries have never been empty, so there is no delay in filling them.
In carrying out the experiment of Johannes Müller I direct that in the expansion of the chest after a deep expiration the mouth should be connected with a mercurial manometer, but of course no air allowed to enter the chest except the few millimetres which he can suck out of the manometer. You can thus see how the experiment is being carried out by the amount and sustention of the nezative pressure in the manometer. Supposing the negative pressure is $80 \mathrm{~mm}$. of mercury, and that the barometer stands at $760 \mathrm{~mm}$., then the intrathoracic pressure will be $760-(80$ plus the elastic tension of the lungs, say, 10) $=670 \mathrm{~mm}$. of mercury, and the pressure on the blood vessels outside the thorax will be $760 \mathrm{~mm}$. Therefore there is a pressure of $90 \mathrm{~mm}$. of mercury driving the blood into the chest, the first effect of which is a storage in the great veins and a good supply to the right side of the heart. The capacity of the lung reservoir is increased, so the immediate effect is a diminished supply to the left side of the heart, and the systemic pulse becomes slower; when the enlarged lung reservoir is filled both sides of the heart are well supplied with blood, and the cardiac action is rather quickened. While this is going on in the chest there is a rapid depletion of the veins and capillaries and a fall in the arterial pressure. If there be a large lung reservoir to fill, the pulse may disappear in many of the superficial arteries for several boats.

In cases of low arterial pressure the arteries are rapidly emptied into the capillaries and veins at their peripheral end, and are not filled at the proximal end, so the pulse disappears. When the lung reservoir overflows, the heart fills up the empty arteries and the pulse again returns even while the chest is fully expanded, and, owing to a good supply of blood to both sides of the heart, its force and frequency are increased. Unlike the Valsalva experiment, the heart at no time stops, and its active movements can be watched under the $x$ rays. The following is a very good example of this experiment carried out on a healthy young man, aged 22 , one of the best mountain climbers in the north of England, and one of the two who made the first ascent into the Devil's Kitchen in North Wales-a feat which in the attempt cost Mr. Hudson his life about two years ago. In the first pulse tracing, after the first two normal pulse waves the chest was fully expanded but no air admitted, and the pulse completely disappeared at the wrist for about thirty beats, and then when he relaxed the inspiratory effort and opened his glottis the pulse immediately reappeared. 
up the extra large lung reservoir, and consequently after the left ventricle had disposed of its immediate supply by two systoles it ceased beating until the lung reservoir overflowed, and then the pulse returned in all the arteries, even with the chest fully expanded. This is well shown in the accompanying pulse tracing (Fig. 4). The chronograph records onefifth second.

I think we have now sufficiently considered the defective filling of the systemic arteries at their central end owing to storage in the enlarged reservoirs of the lungs and other parts of the intratho. racic blood vascular cavity, such as occurs in Müller's experiment; in laryngeal diphtheria and other obstructive lung diseases; in ordinary deep nasal inspiration when there is a good respiratory pump; often in mediastinitis and pleuropericardial adhesions, etc. Where the right ventricle is weak, as in my case of Stokes-Adams disease, ${ }^{21}$ it may take a long time to fill up the large lung reservoir, and so the systemic pulse may disappear so long that the cerebral anaemia jnduces convulsions. We will now consider the emptying of the arteries at their peripheral end into the capillaries and veins.

We have seen how Carson, Barry, Buchanan, Black, Leonard Hill, and myself have shown how the inspiratory suction of the chest and the atmospheric pressure hurries on the circulation in the veins. This relieves the capillaries, and $\mathrm{my}$ tracings ${ }^{12}$ show that in low-tension pulses it has an immediate effect on the arteries. With a weak heart the arteries are not sufficiently filled, and with a good respiratory pump and low tension pulse they are quickly emptied. On the other hand, where there is high blood pressure and a poor respiratory pump, inspiration has scarcely any effect on the pulse. It was held, in explanation of the pulsus paradoxus, that the diminished pressure within the thorax during inspiration retains the blood in, or even sucks it back from, the periphery into the aorta and its intrathoracic branches; but this, in my opinion, is entirely erroneous, as it is impossible to produce a negative pressure within the thorax equal to the positive pressure within the aorta, and so there can be no aspirating effect interfering with the ordinary course of the circulation.

The thorax contains, as I have before said, both an aërial and a blood vascular cavity, and any diminution in the thoracic pressure affects both these cavities. In inspiration both cavities are enlarged, but as they are complementary to one another they will be variously affected by the suction according to the freedom of inflow. In ordinary oral breathing the air enters so freely that there is scarcely any negative pressure, and consequently the circulation receives very little assistance; but in deep nasal breathing, Müller's experiment, etc., there is a great negative pressure, and so the blood rushes on in the veins and capillaries, and the arteries are rapidly drained and the pulse disappears. In my own case I can produce a negative pressure within my thorax of 80 to $90 \mathrm{~mm}$. of mercury, and this causes such a fall in my venous and arterial pressures that my radials are empty for two or three beats of the heart which of course is performing its function all the time. I have discovered that the effect is more pronounced in those who have got not only low blood pressure and soft pliant arteries, but who also have large superficial veins in the arms. In such cases there is greater scope for draining the arterial branch, and so the arteries are more completely emptied, and therefore take longer to fill. The phenomenon is better observed when the arm is depressed than when it is elevated, because, although in the latter case the blood pressure is somewhat lower, the arteries are more contracted, the veins are comparatively empty, and there is not the same opportunity for the drainage of the arteries. On the other hand, in those with high blood pressure or very rigid arteries the respiratory pump can have very little effect on the pulse. In the lower extremities the vessels are more rigid and the blood pressure higher, especially when they are dependent, and the tactile phenomenon can scarcely be observed either in health or disease, but numerous experiments have shown me that this respiratory phenomenon, the so-called pulsus paradoxus, exists in the lower as well as in the upper extremities. Of course in the Valsalva experiment the pulse disappears in all the superficial arteries.

In many cases the pulse is more affected by rapid emptying than by defective filling of the arteries; such is apt to happen in many cases of mediastinitis, extensive pleuropericardial adhesions, moderate left pleural effusion, etc., where the lungs cannot fill up the enlarging thorax or encroach on the heart. In these cases the respiratory pump has a great effect in aspirating the blood from the peripheral vessels into the thorax. In cases of laryngeal diphtheria in elder children with firm chest walls we have a modified Müller's experiment, and here the arterles are rapidly emptied at the periphery, and perhaps badly filled at the centre owing to storage in the lungs and a weak heart. In very young children, where there is almost complete obstruction of the larynx, we do not get the pulsus paradoxus, because when the child attempts to expand its chest, the atmospheric pressure drives in the soft pliant costal cartilages behind which the heart and great blood vessels are situated, and thus the respiratory pump is thrown out of gear. In those cases where there is inspiratory filling of the cervical veins there can be no depletion of the peripheral vessels of the upper extremity, so any irregularity or weakening of the pulse which may occur must be due to lung storage and defective filling. I find that if the venous return from the upper limb is obstructed without shutting off the arterial supply, you cannot obliterate the pulse at the wrist by Müller's experiment, though the pulse does become smaller from the general fall in the blood pressure. Any obstruction of the return from one limb and not from the other would readily explain the unilateral phenomenon recorded by Harris and Gerhardt. In the case recorded by Dr. Harris there was inspiratory swelling of the cervical veins on the right side of the neck, and the pulsus paradoxus was absent in the right radial. Of course there might also be some pressure on one of the subclavian arteries during inspiration.

As I stated and showed in Toronto in my case of StokesAdams disease, and in $\mathrm{my}$ address on the Circulation viewed from the Peripherg: In Müller's experiment, and in cases of the pulsus paradoxus the systole of the left ventricle may be delayed or abolished for one or more beats owing to defect in the diastolic tension in the left ventricle; and the aspirating effect of the thorax causes a sudden emptying of the veins to fill up the vacuum in the chest, and with this removal of the obstruction to the capillary flow there is a simultaneous depletion of the arteries. The pulsus paradoxus is due to one or both of the two following causes, namely, defective filling, or rapid emptying of the arteries. In the former case a weak right heart and a large lung reservoir are important factors, and in the latter a good respiratory pump and low blood pressure are the causal agents.

\section{REFRRENCES.}

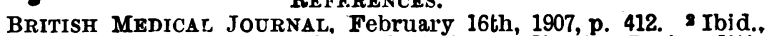

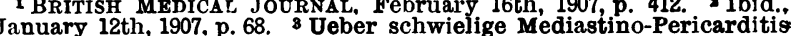
und den paradoxen Puls, Berliner klinische Wochenschrift, 1873. 4 BRITISH MEDICAL JodRNAL, August 25th, 1906, p. 401 . 5 An Inquiry into the Causes of the Motion of the Blood, etc., by Dr. James Carson, 1815. and 1833. ing and upon that function called aboorption, 1826 , by $\mathrm{Dr}$. David Barry. 7 Londom Journal of Medicine, 1850 vol. ii, p. 464. 8 Bauer, Von Ziemssen's Cyclopaedia London, 1876 , vol. vi, p. 649.9 Ibid p 645. 10 Ibid. p. 654. 11 Medical Times and Gazette, January, 1877 . is Liver- 
pool Medico-Chirurgical Journal, July, 1882. 13 BRITISH MEDICAL JOURNAI, July 26th, 1884. 1t Liverpool Medico-Chirurgical Journal, July, 1900, and March, 1901. 15 Charité Annalen, 1877. 16 BRITISH MEDICAL JOURNAL, 1883. ${ }^{17}$ Berliner klinische Wochenschrift. 1883. 18 BRITISH Mrdical Journal, June, 1893. 19 Berliner klinische Wochenechrift, 1897. British Medical Journal, Februal'y 16th, 1907, p. 412.21 Ibid., October 27th, 1906.

\section{THE PULSUS BISFERIENS.}

\section{Bx THOMAS LEWIS, M.B., B.S.,} UNIVERSITY COILEGE, LONDON.

THE observations upon which this contribution is based were made during and since $m y$ term of residence at University College Hospital.

The term "pulsus bisferiens" is now applied to a form of palpably double pulse.* It is described by Broadbent ${ }^{1}$ as having two beats, the second of which is really a reinforcement of a prolonged systole. It is the habit of most writers on the subject to define this type of pulse as one which is double to the finger. If this definition is accepted it follows that every individual observer will have a standard of his own, according to the delicacy of his tactile sense. As this definition is followed in this communication it will be of advantage to add that no case is described as pulsus bisferiens in which the apices of the two waves are separated by a time interval of less than 1 th th of a second, and in which the second wave is not prominent $t^{2}$ Most of the tracings are from pulses in which the time interval is slightly less than $\frac{1}{5}$ th of a second. It has been stated by one observer ${ }^{3}$ that the palsus bisferiens is rare. This is far from being the case. In twenty unselected cases of aortic regurgitation, a double-topped pulse was distinctly appreciable to the finger in thirteen cases. ${ }^{\circ}$ In three it was an obvious phenomenon, and could be seen as well.as felt in the vessels of the arm and neck.

The literature dealing with the pulsus bisferiens from a clinical standpoint is scattered and unconnected. Broadbent ${ }^{1}$ associated it with aortic stenosis and with senile degeneration of the arteries. Steell ${ }^{11}$ found it in connexion with aortic stenosis particularly, but did not consider it pathognomonic of that condition. He stated that a sphygmogram of an ill-developed pulsus bisferiens type is found in aortic incompetence, a conclusion in accord with Mahomed's $s^{8}$ work, and in which the present observations fully concur, though they indicate the frequent presence of a well-developed type also in such conditions. Steell ${ }^{11}$ stated that it is usually accompanied by an ill-developed dicrotic wave, a statement which is by no means free from doubt (cp. Lewis ${ }^{6}$ ). Both D'Espine ${ }^{4}$ and Mackenzie, ${ }^{7}$ associate the double pilse with aortic conditions. So far the position is clear, and it may ke said that almost all writers (cp. Marey, ${ }^{9}$ Galabin, ${ }^{5}$ and others) are agreed that this type of pulse is most frequently associated with disease of the aortic valves. It must be borne in mind that in speaking of the pulsus bisferiens, a character of the pulse rather than its general type is indicated, for there is no distinct dividing line between it and other pulse types; there are all intermediate forms. The transitions to flat-topped, hightension, and anacrotic types are those most commonly seen. It will simplify matters if only that type having a well-marked bisferient character is discussed.

The opinion was originally expressed in this country by Galabin, and is still adhered to in some measure by Mackenzie, ${ }^{7}$ that the splitting of the systolic portion of the tracing into two waves is instrumental in origin; it is impossible to accept this position, on account of the palpability of the waves. ${ }^{6}$ Moreover, Galabin, ${ }^{5}$ who originally attempted to explain the deception of the finger in this respect, was eventually convinced of the double nature of the pulse by its visibility in certain casee. Another point of interest-a point which still remains unsettled-is the fact that the double beat is frequently more easily palpable, and gives a more distinctive tracing on one or other side of the body. Taking the experience of all writers, it cannot be said that it is associated with one side of the body more than with the other; its prominence on one side is of little diagnostic importance unless very marked. The view of Walter Brosdbent ${ }^{2}$ that

* Trousseau applied it to the dicrotic pulse.

+ This statement is based on actual measurements of tracings, in time intervals, employed to check impressions received by palpation. it depends on the arrangement of the aorta and its branches is not tenable, for, if so, it should invariably present more prominence on one side. Steell ${ }^{11}$ said that he had looked in vain for an anatomical explanation in fatal cases. Morrison ${ }^{10}$ was inclined to the view that it is due to varying longitudinal tensions developed in the vessels during the investigation. It is true that it is better shown in tracings taken with the wrist extended, but, as Broadbent ${ }^{1}$ has shown, it is better developed by pressure, and extension of the wrist tends to throw pad pressure on to the vascular contents rather than on to overlying tissues. ${ }^{8}$

Moreover, it is frequently more visible on one side of the neck than the other, and there is sometimes a marked difference in tracings from the two superficial temporal arteries, where longitudina] tension can play little or no part (Fig. 1). Again, in cases in which it occurs on one side it is often impossible to obtain it on the other by rearranging the limb. In a previous communication it was pointed out that the height of the second wave of the pulsus bisferiens depends on the degree of arteriosclerosis present. Differences on the two sides are possibly due to differences in the expansibility of the two vessels. The association of arterio-sclerosis is so close that it is probable that in aortic cases it forms an essential factor to its appearance at the wrist. I venture to place certain cases which will be described later, and which are unassociated with sclerosis, in a different etiological category.

Dealing with the first or generally-recognized type (Figs. 1 to 4), it may be said to occur in those cases in
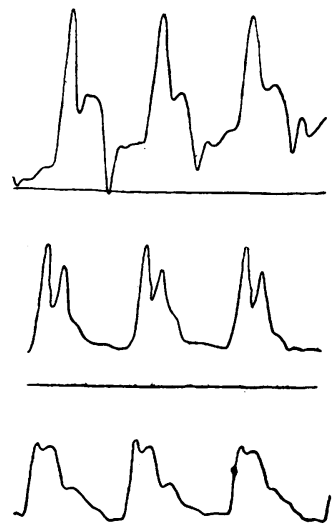

Fig. 1.-From above down, cardiogram, left and right superficial temporal arteries.

which there is great enlargement of the left heart, associated with or caused by disease of the aortic valves or larger arteries. Attempts have been made to draw conclusions from it as to the relative degree of hypertrophy and dilatation, ${ }^{3}$ but the relationship appears to be inconstant. Some writers associate it with poor compensation, others with improved compensation. Steell ${ }^{11}$ noticed its development after the administration of digitalis. In more than one case I have noticed it for a day or two without further change in physical signs. In one case it developed in conjunction with some symptoms of cardiac distref $\mathrm{s}$. In the literature three morbid conditions are commonly described in connexion with it: a greatly enlarged heart, $\ddagger$ pericardial adhesions or excess of fluid, and lastly ballooning of the aorta. It would appear that in aortic cases it is best developed in those cases where with great enlargement of the left heart compensation, though for the moment sufficient, is in a state of instability. In Fig. 1 tracings are shown from the H.A.B $\S$ and superficial temporal arteries of a patient aged 30, with free aortic regurgitation, poor compensation, great cardiac enlargement, and a fair degree of arterio sclerosis.

t The association of this pulse with great left cardiac enlargement is suggested by its frequency in aortic disease.

suggested by its frequency in aortic disease. Al that a fairer comparison might be instituted between them and the corresponding sphygmograms. 